\title{
Enhanced Light Trapping in Thin Film Solar Cells Using a Plasmonic Fishnet Structure
}

\author{
Sayan Seal, Vinay Budhraja, Liming Ji, and Vasundara V. Varadan \\ Department of Electrical Engineering, University of Arkansas, Fayetteville, AR 72701, USA \\ Correspondence should be addressed to Sayan Seal; seal@uark.edu
}

Received 18 September 2014; Revised 11 January 2015; Accepted 11 January 2015

Academic Editor: Ahmad Umar

Copyright (C) 2015 Sayan Seal et al. This is an open access article distributed under the Creative Commons Attribution License, which permits unrestricted use, distribution, and reproduction in any medium, provided the original work is properly cited.

Incorporating plasmonic structures into the back spacer layer of thin film solar cells (TFSCs) is an efficient way to improve their performance. The fishnet structure is used to enhance light trapping. Unlike other previously suggested discrete plasmonic particles, the fishnet is an electrically connected wire mesh that does not result in light field localization, which leads to high absorption losses. The design was verified experimentally. A silver fishnet structure was fabricated using electron beam lithography (EBL) and thermal evaporation. The final fabricated structure optically resembles a TFSC. The results predicted by numerical simulations were reproduced experimentally on a fabricated sample. We show that light absorption in the a-Si absorber layer is enhanced by a factor of 10.6 at the design wavelength of $690 \mathrm{~nm}$ due to the presence of the fishnet structure. Furthermore, the total absorption over all wavelengths was increased by a factor of 3.2. The short-circuit current of the TFSC was increased by $30 \%$ as a result of including the fishnet.

\section{Introduction}

Amorphous silicon thin film solar cells (TFSCs) are an attractive alternative to crystalline silicon wafer solar cells. The material required to produce a TFSC is only a fraction of that used to make a wafer solar cell. Other advantages include low cost processing and easy deposition at comparatively lower temperatures on a variety of substrates [1-3], including flexible substrates. The thickness of TFSCs is typically a fraction of that of wafer solar cells, but the absorption of light in the thin film has to be comparable to a wafer cell in order to be competitive in the market today. This can be achieved by increasing the light path in the absorber layer through the scattering of the incoming light.

There are various techniques used in the processing of TFSCs to enhance absorption. The simplest technique would be to use a metallic back reflector to double the optical path length of incoming light. Extending this concept further, roughness was introduced to the geometry of the metal back reflector or the TCO layers to enhance light absorption by diffuse scattering [4-9]. Theoretical analysis of rough surfaces was shown theoretically by Yablonovitch in 1982 [10, 11]. Random roughness on material surfaces has been widely used in the industry, but the enhancement of light absorption using this method is limited. Hence, efforts have been made to manipulate the geometry of the back reflector geometry.

Photonic back reflectors $[12,13]$ and diffraction gratings $[14,15]$ were good propositions that utilized planar structures to aid light absorption. Shaping a solar cell itself into a geometry, such as nanodome, can also significantly improve the efficiency of a TFSC $[16,17]$.

Another approach to cut down the thickness of the scatterers was realized by using metal nanoparticles embedded within the front or back spacer layer. Several front surface designs were proposed which resulted in an improvement in the performance of thin film solar cells [18-26]. Studies showed that for red shifting particle resonances, larger particles were required that degraded short wavelength performance of the TFSC. This exposes a major drawback of plasmonic nanoparticle based designs. For enhancement at longer wavelengths, larger particle sizes are required, which will obstruct incoming light [20] at shorter wavelengths. In a study by Pala et al. [27], periodic plasmonic particles exceeding a critical dimension of $100 \mathrm{~nm}$ were shown to act like reflectors. Research efforts are being made on moving plasmonic structures to the back spacer layer in thin film solar cells based on the fact that front surface plasmonics tend to suppress photocurrent generation at short wavelengths [28]. 


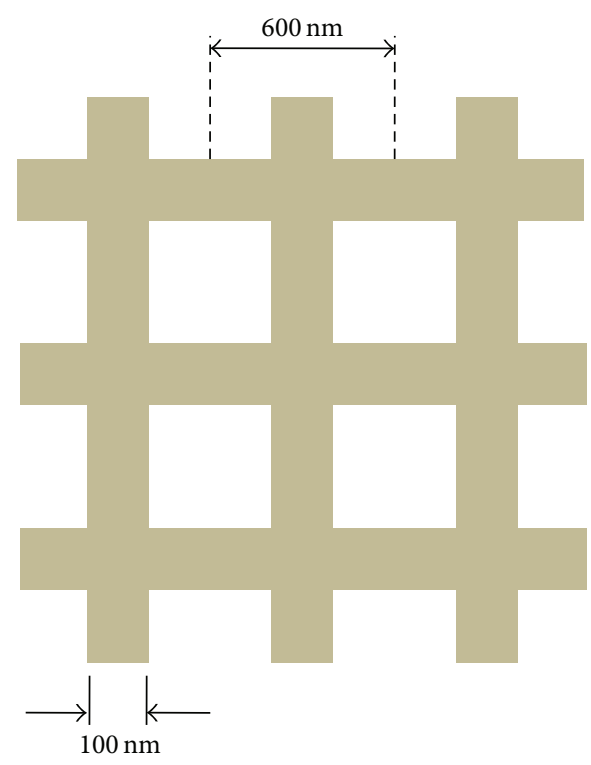

(a) Fishnet specifications

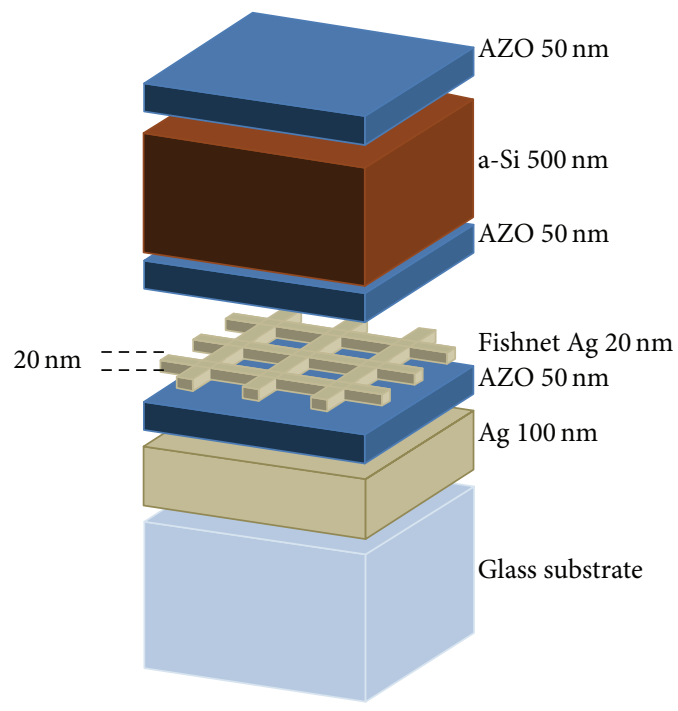

(b) Schematic of the fishnet TFSC

FIGURE 1: Optimized model for the TFSC with a fishnet in the back spacer layer.

Also, using isolated nanoparticles results in high electric field localization around the particle itself, which leads to high absorption losses. This is because metals behave like dielectric materials with high attenuation coefficients at optical frequencies. Optical waves penetrate into metals and get attenuated. The reduced photon energy is converted into heat as undesired loss.

The plasmonic fishnet structure proposed by Ji and Varadan [29] is an electrically connected, plasmonic structure whose geometry can be optimized to realize absorption enhancement in the a-Si layer of a TFSC at a particular target wavelength. It is a planar structure embedded within the back spacer of a TFSC. In a-Si, the absorption is very low for wavelengths $\sim 700 \mathrm{~nm}$ near the bandgap. Hence, the resonance frequency of the fishnet was designed to enhance absorption in this region. The fishnet structure has two distinct advantages compared to other particulate or discrete plasmonic structures. (1) The field localization in the fishnet plasmonic structure is much smaller in comparison. (2) It enhances the conductivity of the back spacer and can also serve as the back electrode. The ability of this structure to enhance the absorption and $J_{\mathrm{SC}}$ in TFSCs was shown by numerical simulation in the past [29]. Following this design very closely, a plasmonic fishnet structure was embedded within the back spacer of a TFSC structure. The incoming light is scattered into the absorber layer and also trapped by total internal reflection from the top interface. This particular fishnet provides a plasmonic resonance at the bandgap of a-Si, which corresponds to a wavelength of around $690 \mathrm{~nm}$.

\section{Materials and Methods}

2.1. Design and Fabrication of the Fishnet TFSC. The design of the fishnet TFSC is based on the previous work which was referred to earlier [29]. The only differences in the device dimensions arose as a result of changing the choice of metal for both the fishnet and the back electrode to silver instead of gold. All other materials were kept the same. The optimized design is shown in Figure 1.

Figure 1(b) shows the complete structure of the fabricated sample, which optically resembles a TFSC. With this set of dimensions, the fishnet structure was expected to resonate near $700 \mathrm{~nm}$, which is the bandgap of a-Si. The bandgap region of a-Si has a very low absorption coefficient; hence light absorption enhancement is most required at this wavelength. Since this is a single layer fishnet, there is no interlayer magnetic coupling that exists in multilayer fishnet structure [30]. Instead, only electrical resonance will be generated, resulting in an oscillating current in the metallic structure. This oscillating current is the result of photon-electron energy conversion and then will scatter photons back into the a-Si layer.

A $100 \mathrm{~nm}$ layer of silver was deposited on a glass substrate by thermal evaporation, using $\mathrm{Cr}$ as an adhesion layer. Silver thin films were found to disintegrate easily in the absence of the Cr layer. Al-doped zinc oxide (AZO) was then deposited on the top of the silver layer using atomic layer deposition (ALD). The fishnet structure was then fabricated using electron beam lithography, thermal evaporation and subsequent lift-off using acetone. A $7 \mathrm{~nm}$ thin film of $\mathrm{Cr}$ was required to ensure proper adhesion of the silver to the AZO during this step. This was followed by another AZO deposition step to cover the fishnet completely, thus embedding the fishnet within the AZO back spacer. a-Si was deposited over this using Plasma Enhanced Chemical Vapor Deposition under $900 \mathrm{mTorr}, 50 \mathrm{~W}$ for 17 minutes, to give a $500 \mathrm{~nm}$ layer. Finally, a $50 \mathrm{~nm}$ AZO front spacer layer was deposited to help reduce reflection from the top of the TFSC. The pattern was fabricated within an area of $1.048 \mathrm{~mm} \times 1.048 \mathrm{~mm}$. 
2.2. Measurement of Total Absorption. After a repeatable process was established for fabricating the solar cell, the absorption was measured. As a control experiment, a TFSC without a fishnet was used. However, the thicknesses and materials of all layers were kept unaltered. The samples were characterized optically using spectroscopic ellipsometry. The VASE ellipsometer from the J. A. Woollam Company was used to take measurements. The angle of incidence was kept fixed at $15^{\circ}$. Ideally measurements should have been taken at normal incidence, but $15^{\circ}$ was the minimum achievable angle for the ellipsometer used for this measurement. Measurements were taken for three different polarization states-p-polarized (electric field vector along the plane of incidence), s-polarized (electric field perpendicular to the plane of incidence), and u-polarized (unpolarized). The plane of polarization for unpolarized light was set at $45^{\circ}$, but the result would be qualitatively similar for any arbitrary angle. This was important in determining whether the fishnet behaves isotropically with respect to light with different polarization states.

The ellipsometer cannot measure absorption directly. However, it can measure the coefficients of reflection $(R)$ and transmission $(T)$. However, because the back reflector $T$ is negligible, this claim was verified experimentally, and the transmission is shown plotted as a function of frequency in Figure 4. Hence the coefficient of absorption $(A)$ simply becomes

$$
A=1-R \text {. }
$$

Also, it must be noted that specular reflection and direct transmission were measured.

2.3. Calculation of the Short-Circuit Current Density $\left(J_{S C}\right)$. Equation (1) gives a measure of the total power absorbed by the whole solar cell. This is not necessarily equal to the power absorbed by the a-Si layer, because it includes losses occurring within the whole solar cell structure. It is important to estimate the power absorbed within the a-Si layer while calculating the $J_{\mathrm{SC}}$, because this absorption gives rise to electronhole pairs. At each wavelength, the fraction of light absorbed in the a-Si layer $\left(f_{\mathrm{a}-\mathrm{Si}}\right)$ was calculated from the simulation results. From this estimate, the actual absorption in a-Si in the fabricated sample was calculated using the following relation:

$$
A_{\mathrm{a}-\mathrm{Si}}=f_{\mathrm{a}-\mathrm{Si}} \times A .
$$

Once the absorption in a-Si $\left(A_{\mathrm{a}-\mathrm{Si}}\right)$ was calculated using (2), the $J_{\mathrm{SC}}$ was estimated using the following relation:

$$
J_{\mathrm{SC}}=\int_{\lambda} e A_{\mathrm{a}-\mathrm{Si}}(\lambda) \Phi_{0}(\lambda) d \lambda
$$

where, $\Phi_{0}$ is the wavelength dependent solar photon flux density and $e$ is the electronic charge.

2.4. Comparison of Experimental Results and Numerical Simulation. The model of the fishnet TFSC in Ansoft HFSS was modified based on certain observations after the fabrication process was completed. It was noted that (1) the AZO layer covering the fishnet was not flat, it had undulations above the area covered by the fishnet, and (2) the optical constants of the materials used for fabrication were not identical to the values used in simulation. Although the differences were within 5\%, it was enough to influence the absorption profile obtained from simulations. Also the $7 \mathrm{~nm} \mathrm{Cr}$ adhesion layer below the silver fishnet had to be accounted for.

The existence of bumps above the fishnet was verified using atomic force microscopy (AFM), and the model was edited accordingly. All materials used to fabricate the fishnet TFSC were characterized using spectroscopic ellipsometry. These values served as the inputs for the simulation process.

\section{Results and Discussions}

3.1. Fabrication Results for the Fishnet TFSC. Figure 2 shows the SEM image of the fabricated fishnet structure. The pattern is uniform and matches the design specifications well. The line width achieved was $100 \mathrm{~nm}$ on an average, with an average pitch size of $598.5 \mathrm{~nm}$. The pattern was uniform throughout the patterned area, as can be seen from Figure 2(b).

Without the Cr adhesion layer, the silver fishnet could not adhere to the AZO layer. It was found to disintegrate during the lift-off process. The result from one such process is shown in Figure 3.

3.2. Measurement of Absorption of the TFSC with and without the Fishnet. Before measuring the absorption, the applicability of (1) had been verified. The direct transmission was measured to be a little over $0.35 \%$ at the most, especially at wavelengths beyond $700 \mathrm{~nm}$ which contribute minimally toward carrier generation (Figure 4). The average transmission over the entire wavelength range was about $0.08 \%$.

The total absorption with and without fishnet was plotted and is shown in Figure 5(a). A 10.6x increase in total absorption was observed at a wavelength of $690 \mathrm{~nm}$. Moreover, the performance at lower wavelengths was also enhanced as a result of including the fishnet. It is worth noting that the enhancement at lower wavelengths is not a plasmonic effect but is a consequence of scattering at shorter wavelengths. The fishnet is principally a light scatterer. The scattering is enhanced at the plasmonic resonance frequency. However for lower wavelengths, a significant amount of scattering always occurs because scattering is proportional to the inverse of wavelength. Even though scattering exists at lower wavelengths, it is not due to the plasmonic effect associated with the fishnet-it occurs because light at a low wavelength is incident on a corrugated surface.

This is a distinct advantage of the fishnet as compared to other discrete plasmonic structures that become electrically very large at shorter wavelengths. The average absorption enhancement due to the fishnet across the entire wavelength range was in fact $3.2 \mathrm{x}$.

Figure 5(b) shows measurements taken for four different samples. This step was important to verify the repeatability of both the fabrication process, as well as the characterization technique. We find very good correlation in the absorption spectrum obtained from different samples. It can be seen 


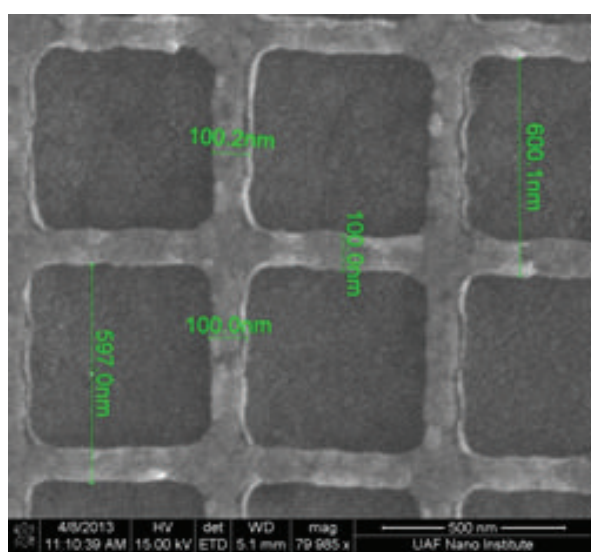

(a) Fishnet structure

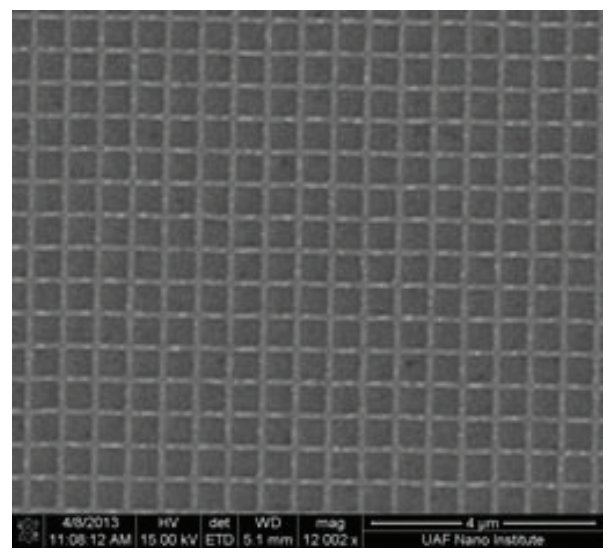

(b) Pattern uniform over the entire area

FIGURE 2: SEM image of the fabricated fishnet structure.

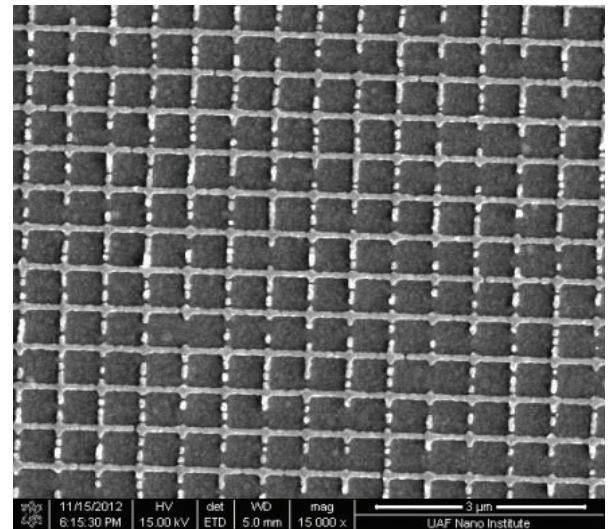

FIGURE 3: Illustration of inadequate adhesion without Cr underlayer.

from Figure 5(c) that the absorption spectrum is nearly independent of the state of polarization. Thus, the performance of the fishnet is not polarization limited; light of different polarizations will contribute equally toward the enhancement of scattering.

For plasmonic designs, it is not a valid assumption that the total light absorbed by the sample is equal to the light absorbed within the a-Si layer. Note that it is the absorption within the a-Si layer that gives rise to electron-hole pair generation. The total absorption also includes the loss in the metallic plasmonic structure and hence may be a misleading interpretation of absorption enhancement. Hence it is imperative to analyze the layer-wise absorption in the TFSC and verify that a majority of the absorption occurs within the a-Si layer. Figure 6 shows the results of this analysis, which was obtained through a full wave EM simulation of the fishnet TFSC in Ansoft HFSS. It can be observed that at plasmonic resonance only $16 \%$ of the power is dissipated in the fishnet and $1 \%$ in the spacer layers and the back electrode combined. $83 \%$ of the power is absorbed by the a-Si layer.

From Figure 6 we can also observe two absorption peaks of the fishnet around $700 \mathrm{~nm}$. These correspond to the plasmonic resonance frequencies of the fishnet structure and are

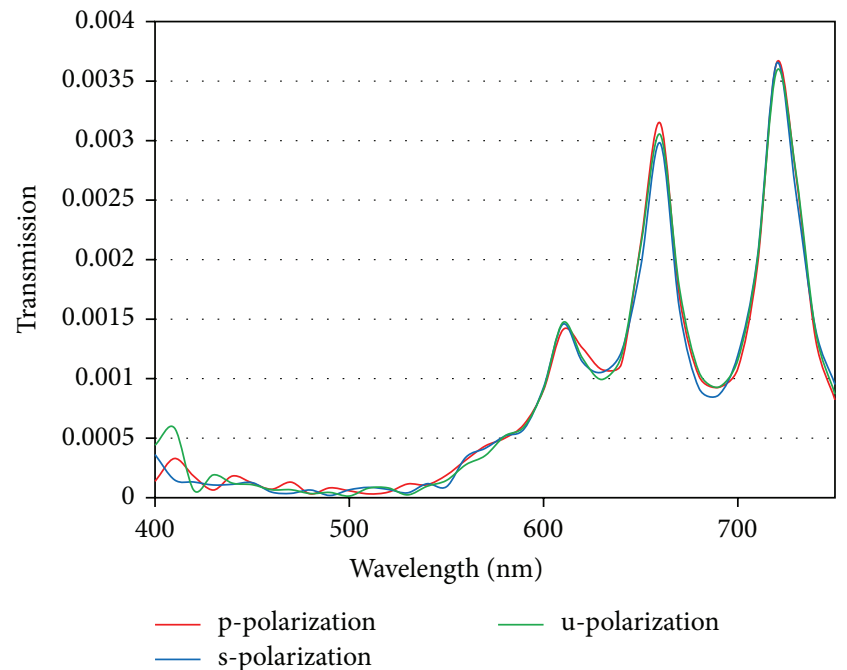

FIgURE 4: Transmission through the fishnet TFSC.

indicative of the absorption loss in a plasmonic structure at the plasmonic resonance frequencies. For a good plasmonic design, these losses should be minimized. Even despite this loss, there is an overall absorption enhancement as can be seen from Figure 5(a). It must be noted, however, that the resonance frequency at $730 \mathrm{~nm}$ does not contribute to absorption enhancement since the absorption in a-Si is practically zero beyond $700 \mathrm{~nm}$.

To further investigate the absorption enhancement at the resonance frequency, the electric field pattern was computed in HFSS. Figure 7 presents the electric field plot within the aSi layer with and without the fishnet at plasmonic resonance. It can be clearly observed that there are several localized hotspots within the a-Si layer. The relative electric field in these hotspots is enhanced by a factor of about 250 as a result of the fishnet. Moreover, even aside from the hotspots, the electric field in the less intense regions of the fishnet TFSC is also greater than that of the planar TFSC by at least two orders of magnitude. 


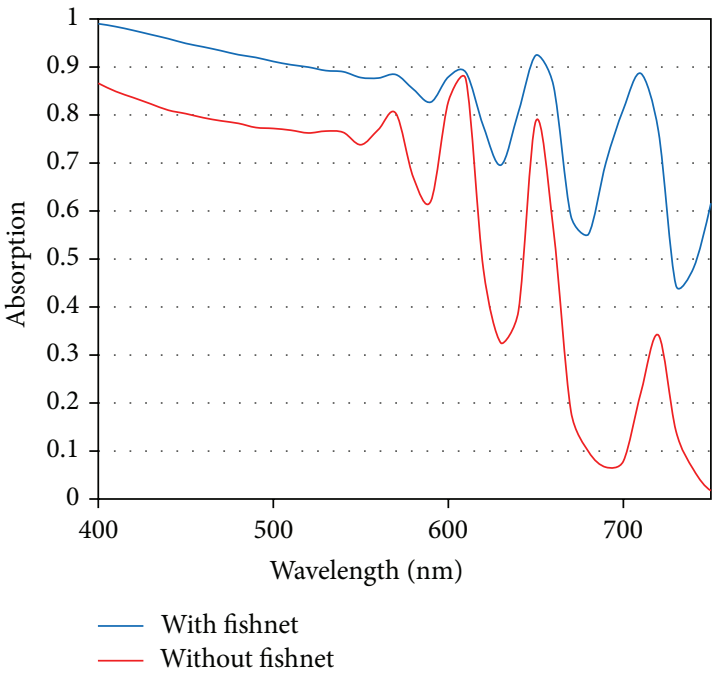

(a) Measured total absorption with and without the fishnet

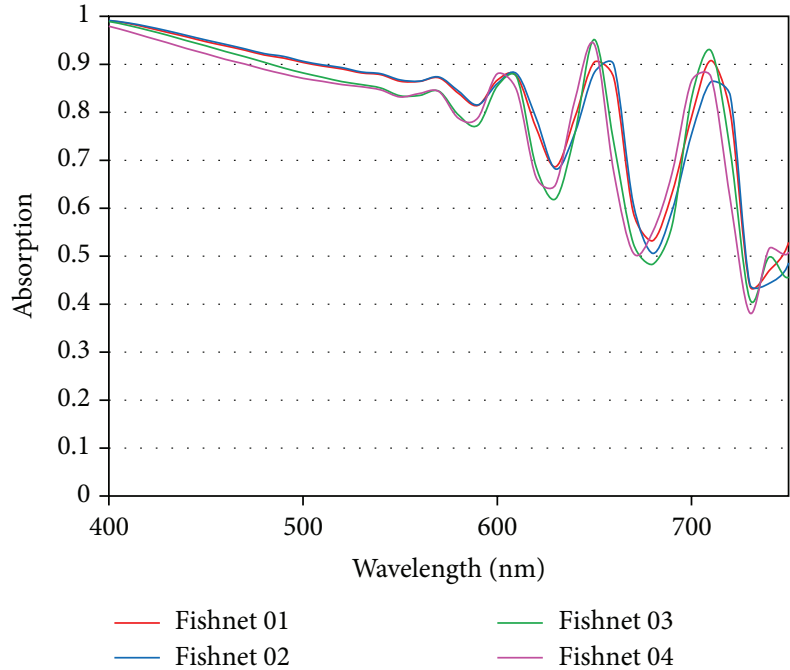

(b) Measured total absorption for four different samples

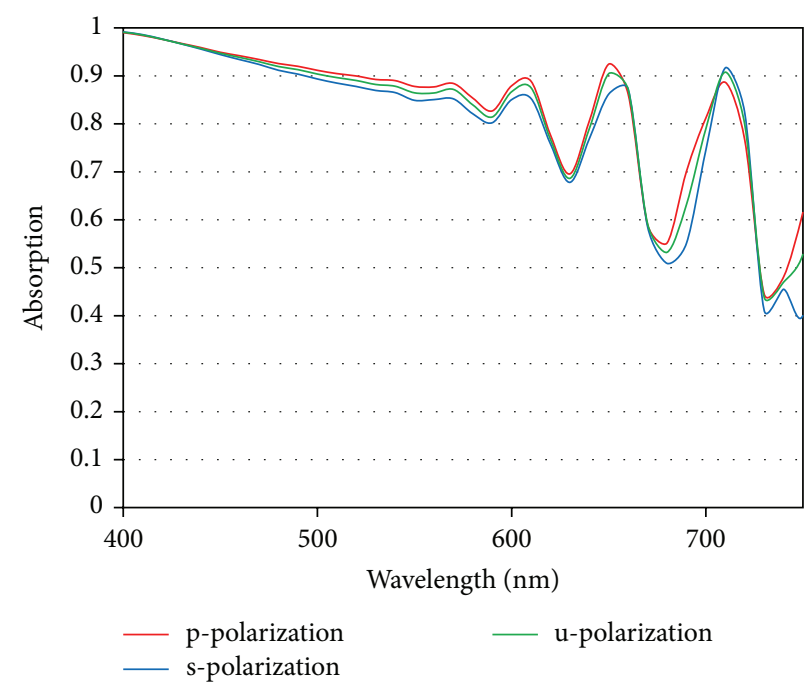

(c) Measured total absorption for different polarizations

FIgURE 5: Measurement of total absorption.

TABLE 1: Calculated $J_{\text {SC }}$ for TFSCs with and without the fishnet structure.

\begin{tabular}{lcc}
\hline S. number & TFSC & $J_{\text {SC }}\left(\mathrm{mA} / \mathrm{cm}^{2}\right)$ \\
\hline 1 & Without fishnet & 11.751 \\
2 & With fishnet 01 & 15.6553 \\
3 & With fishnet 02 & 15.6775 \\
4 & With fishnet 03 & 14.9136 \\
5 & With fishnet 04 & 14.9464 \\
\hline
\end{tabular}

3.3. Calculation of Short-Circuit Current Density $\left(J_{S C}\right)$. Table 1 shows the $J_{\text {SC }}$ calculated using the absorption in the a-Si layer only. There was a $30 \%$ increase on an average in $J_{\text {SC }}$ as a result of including the fishnet. The calculation does not take the diffusion length of the carriers since the thickness of the active region is very small in comparison. The solar irradiation spectrum is accounted for in the calculations.
It is worth noting that the fishnet structure can serve as a back electrode for the solar cell as well. It is worth noting that the fishnet structure can serve as a back electrode for the solar cell as well.

3.4. Comparison between Experimental and Simulated Results. A numerical model for the fabricated fishnet TFSC was created in Ansoft's HFSS. If a fair comparison was to be made, the model had to be upgraded to incorporate some details pertaining to the fabrication process. The schematic in Figure 8(a) shows that a $27 \mathrm{~nm}$ bump was modeled in the AZO layer covering the fishnet. The existence of this bump was verified using atomic force microscopy (AFM) measurements (Figure 8(b)). In addition the $7 \mathrm{~nm} \mathrm{Cr}$ adhesion layer below the fishnet was also included in the model. All optical constants of the materials used to fabricate the TFSC were characterized using spectroscopic ellipsometry 


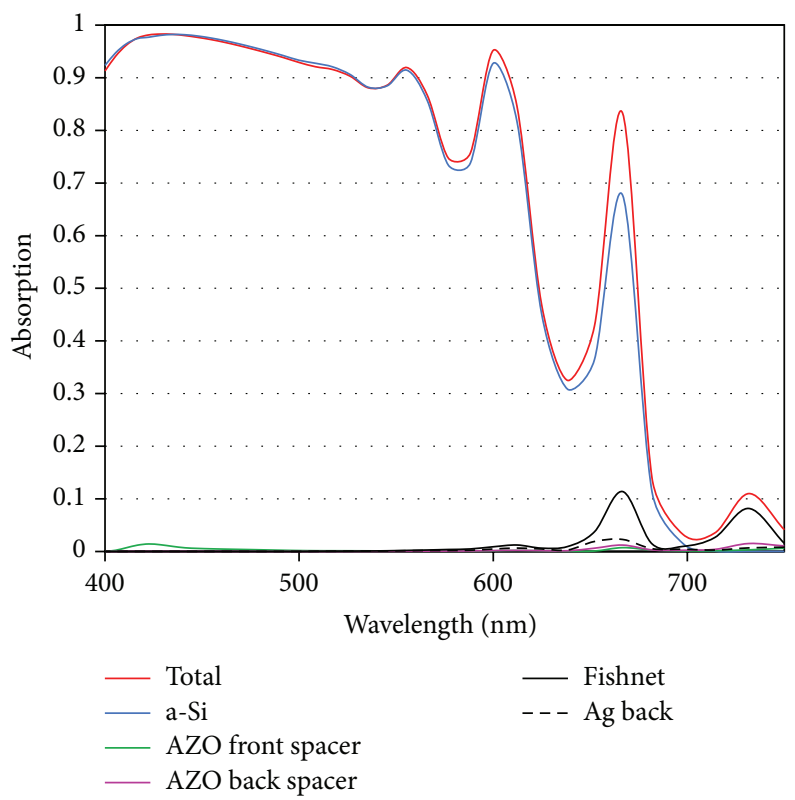

FIGURE 6: Layer-wise absorption analysis.

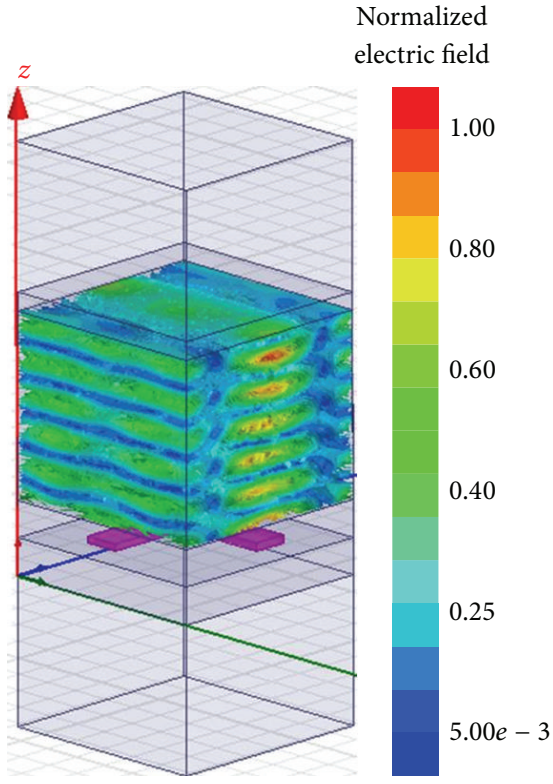

(a)

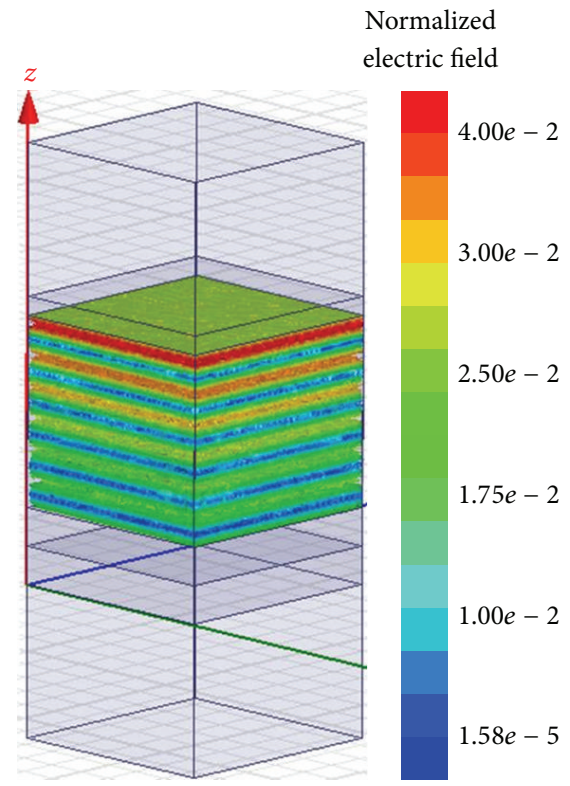

(b)

FIGURE 7: Electric field plot with (b) and without (a) the fishnet.

in the wavelength range of interest. These were the values used for the numerical simulation, not reference values from literature. Optical constants differ depending on the purity of materials and the equipment used to deposit these materials. Even minor differences in the optical properties can cause considerable changes in the absorption value estimated by HFSS. Figure 8(c) shows the comparison between the numerical simulation and the absorption obtained experimentally.

The disparity in the result can be attributed to two main factors: (1) the effect of interface roughness between the layers of the TFSC was not modeled and (2) the simulation was carried out for normally incident light whereas the experiment was done for light incident at a $15^{\circ}$ angle, since this was the minimum angle of incidence attainable by the ellipsometer.

\section{Conclusion}

The benefits of incorporating a plasmonic fishnet structure in the back spacer of TFSCs have been demonstrated theoretically in the past. This paper provides experimental proof of this claim. Numerical simulations predict the enhancement 


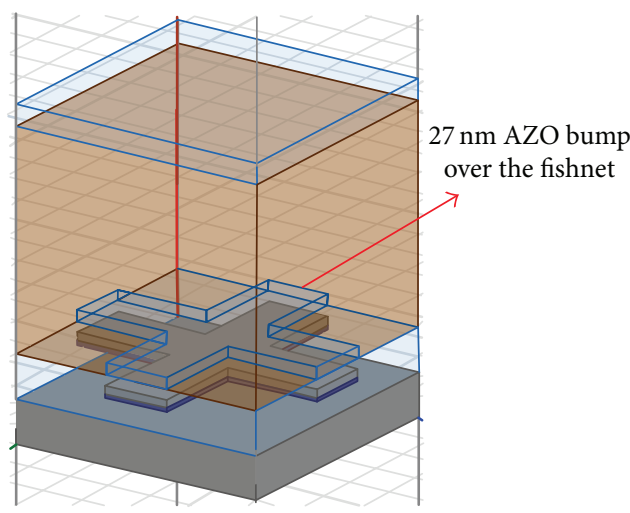

(a) HFSS model

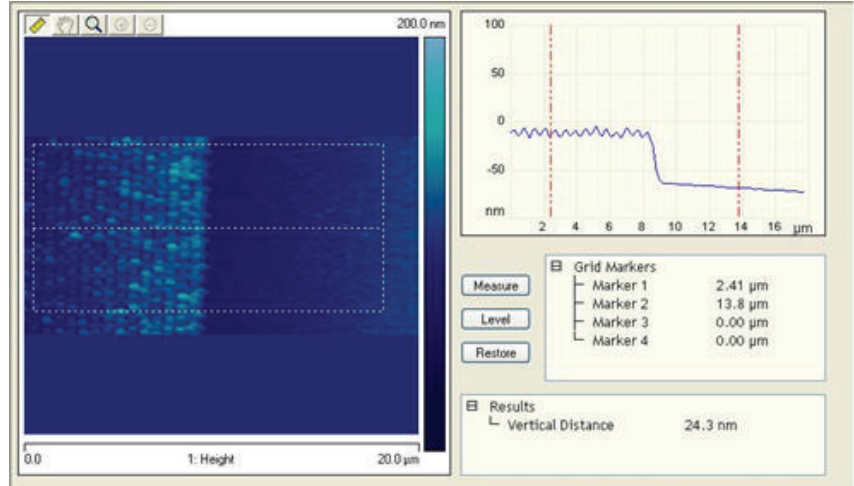

(b) AFM results showing a $24.3 \mathrm{~nm}$ bump

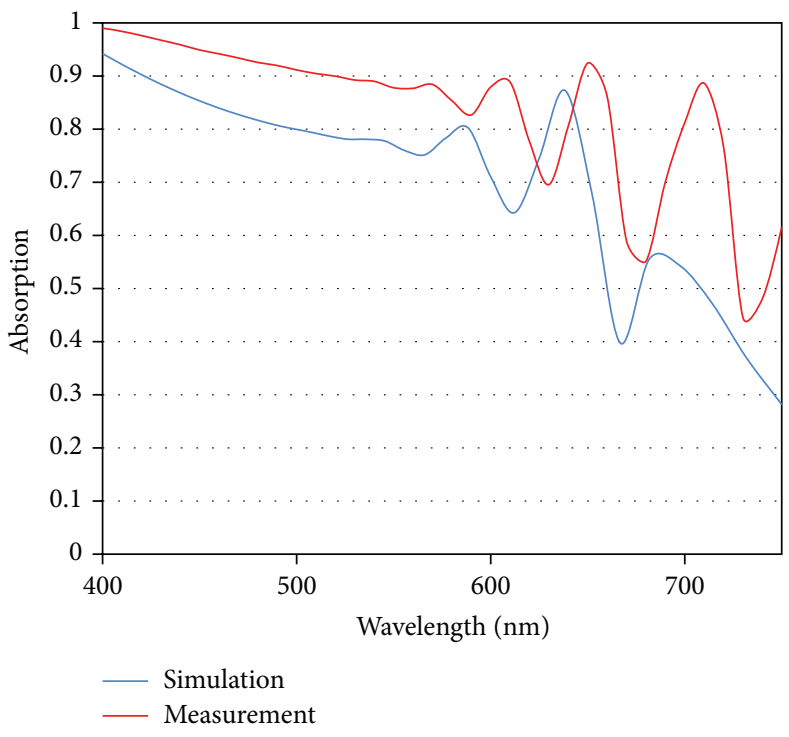

(c) Comparison between theory and experiment

FIgURE 8: Modeling results.

of absorption at a wavelength of about $670 \mathrm{~nm}$. It was also shown from HFSS simulations that the trapped light is absorbed mostly by the a-Si layer in the TFSC. A complete process for fabricating the fishnet structure was developed as seen from SEM pictures. The total absorption of the TFSC is enhanced by a factor of 10.6 at $690 \mathrm{~nm}$ as a result of including the fishnet in the back spacer layer. Furthermore, the average enhancement across the considered range of wavelengths was found to increase by a factor of 3.2 resulting in a $30 \%$ increase in $J_{\mathrm{SC}}$. This is supplemented by the fact that the fishnet structure can also serve as the back electrode. It was also shown that agreement between simulation and experimental data can be greatly improved by accurate characterization of the constituent materials and by adding appropriate structural modifications. The fishnet is just under $30 \mathrm{~nm}$ thick-this makes it possible to use ultra-thin layers of semiconductor material, while maintaining an electrical output comparable to wafer based cells. This also makes it a promising technology to use for flexible solar panels. Thus, if properly designed, this structure has great potential for the enhancement of absorption in TFSCs in general.

\section{Conflict of Interests}

The authors declare that there is no conflict of interests regarding the publication of this paper.

\section{Acknowledgments}

The authors acknowledge the research support provided by the National Science Foundation under EPS-1003970. They also acknowledge the collaboration with Dr. J. Cui, University of Arkansas at Little Rock, for AZO deposition.

\section{References}

[1] A. Shah, P. Torres, R. Tscharner, N. Wyrsch, and H. Keppner, "Photovoltaic technology: the case for thin-film solar cells," Science, vol. 285, no. 5428, pp. 692-698, 1999.

[2] A. V. Shah, H. Schade, M. Vanecek et al., "Thin-film silicon solar cell technology," Progress in Photovoltaics: Research and Applications, vol. 12, no. 2-3, pp. 113-142, 2004.

[3] S. Hegedus, "Thin film solar modules: the low cost, high throughput and versatile alternative to Si wafers," Progress in 
Photovoltaics: Research and Applications, vol. 14, no. 5, pp. 393411, 2006.

[4] O. Kluth, B. Rech, L. Houben et al., "Texture etched $\mathrm{ZnO}: \mathrm{Al}$ coated glass substrates for silicon based thin film solar cells," Thin Solid Films, vol. 351, no. 1-2, pp. 247-253, 1999.

[5] J. Müller, B. Rech, J. Springer, and M. Vanecek, “TCO and light trapping in silicon thin film solar cells," Solar Energy, vol. 77, no. 6, pp. 917-930, 2004.

[6] J. Zhao, A. Wang, M. A. Green, and F. Ferrazza, "19.8\% efficient 'honeycomb' textured multicrystalline and $24.4 \%$ monocrystalline silicon solar cells," Applied Physics Letters, vol. 73, no. 14, pp. 1991-1993, 1998.

[7] J. Hüpkes, B. Rech, O. Kluth et al., "Surface textured MFsputtered $\mathrm{ZnO}$ films for microcrystalline silicon-based thinfilm solar cells," Solar Energy Materials and Solar Cells, vol. 90, no. 18-19, pp. 3054-3060, 2006.

[8] J. Springer, B. Rech, W. Reetz, J. Müller, and M. Vanecek, "Light trapping and optical losses in microcrystalline silicon pin solar cells deposited on surface-textured glass/ZnO substrates," Solar Energy Materials and Solar Cells, vol. 85, no. 1, pp. 1-11, 2005.

[9] Z. R. Abrams, A. Niv, and X. Zhang, "Solar energy enhancement using down-converting particles: a rigorous approach," Journal of Applied Physics, vol. 109, no. 11, Article ID 114905, 2011.

[10] E. Yablonovitch, "Statistical ray optics," Journal of the Optical Society of America, vol. 72, no. 7, pp. 899-907, 1982.

[11] E. Yablonovitch and G. D. Cody, "Intensity enhancement in textured optical sheets for solar cells," IEEE Transactions on Electron Devices, vol. 29, no. 2, pp. 300-305, 1982.

[12] P. Bermel, C. Luo, L. Zeng, L. C. Kimerling, and J. D. Joannopoulos, "Improving thin-film crystalline silicon solar cell efficiencies with photonic crystals," Optics Express, vol. 15, no. 25, pp. 16986-17000, 2007.

[13] D. Zhou and R. Biswas, "Photonic crystal enhanced lighttrapping in thin film solar cells," Journal of Applied Physics, vol. 103, no. 9, Article ID 093102, 2008.

[14] P. Sheng, A. N. Bloch, and R. S. Stepleman, "Wavelength-selective absorption enhancement in thin-film solar cells," Applied Physics Letters, vol. 43, no. 6, pp. 579-581, 1983.

[15] J. G. Mutitu, S. Shi, C. Chen et al., "Thin film silicon solar cell design based on photonic crystal and diffractive grating structures," Optics Express, vol. 16, no. 19, pp. 15238-15248, 2008.

[16] J. Zhu, C.-M. Hsu, Z. Yu, S. Fan, and Y. Cui, "Nanodome solar cells with efficient light management and self-cleaning," Nano Letters, vol. 10, no. 6, pp. 1979-1984, 2010.

[17] W. J. Nam, L. Ji, T. L. Benanti et al., "Incorporation of a light and carrier collection management nano-element array into superstrate a-Si:H solar cells," Applied Physics Letters, vol. 99, no. 7, Article ID 073113, 2011.

[18] K. R. Catchpole and A. Polman, "Plasmonic solar cells," Optics Express, vol. 16, no. 26, pp. 21793-21800, 2008.

[19] D. Derkacs, S. H. Lim, P. Matheu, W. Mar, and E. T. Yu, "Improved performance of amorphous silicon solar cells via scattering from surface plasmon polaritons in nearby metallic nanoparticles," Applied Physics Letters, vol. 89, Article ID 093103, 2006.

[20] S. Pillai, K. R. Catchpole, T. Trupke, and M. A. Green, "Surface plasmon enhanced silicon solar cells," Journal of Applied Physics, vol. 101, no. 9, Article ID 093105, 2007.

[21] D. M. Schaadt, B. Feng, and E. T. Yu, "Enhanced semiconductor optical absorption via surface plasmon excitation in metal nanoparticles," Applied Physics Letters, vol. 86, no. 6, Article ID 063106, 2005.
[22] V. E. Ferry, M. A. Verschuuren, H. B. T. Li et al., "Light trapping in ultrathin plasmonic solar cells," Optics Express, vol. 18, no. 13, pp. A237-A245, 2010.

[23] V. E. Ferry, L. A. Sweatlock, D. Pacifici, and H. A. Atwater, "Plasmonic nanostructure design for efficient light coupling into solar cells," Nano Letters, vol. 8, no. 12, pp. 4391-4397, 2008.

[24] H. A. Atwater and A. Polman, "Plasmonics for improved photovoltaic devices," Nature Materials, vol. 9, no. 3, pp. 205-213, 2010.

[25] K. Nakayama, K. Tanabe, and H. A. Atwater, "Plasmonic nanoparticle enhanced light absorption in GaAs solar cells," Applied Physics Letters, vol. 93, no. 12, Article ID 121904, 2008.

[26] J. A. Schuller, E. S. Barnard, W. S. Cai, Y. C. Jun, J. S. White, and M. L. Brongersma, "Plasmonics for extreme light concentration and manipulation," Nature Materials, vol. 9, pp. 193-204, 2010.

[27] R. A. Pala, J. White, E. Barnard, J. Liu, and M. L. Brongersma, "Design of plasmonic thin-film solar cells with broadband absorption enhancements," Advanced Materials, vol. 21, no. 34, pp. 3504-3509, 2009.

[28] H. Tan, R. Santbergen, A. H. M. Smets, and M. Zeman, "Plasmonic light trapping in thin-film silicon solar cells with improved self-assembled silver nanoparticles," Nano Letters, vol. 12, no. 8, pp. 4070-4076, 2012.

[29] L. Ji and V. V. Varadan, "Fishnet metastructure for efficiency enhancement of a thin film solar cell," Journal of Applied Physics, vol. 110, Article ID 043114, 2011.

[30] J. Valentine, S. Zhang, T. Zentgraf et al., "Three-dimensional optical metamaterial with a negative refractive index," Nature, vol. 455, no. 7211, pp. 376-379, 2008. 

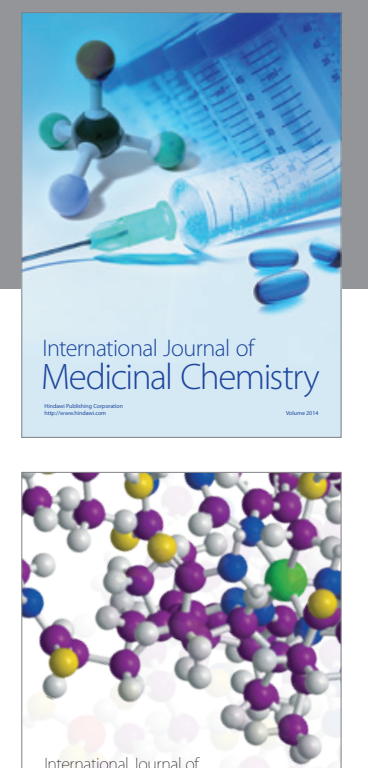

\section{Carbohydrate} Chemistry

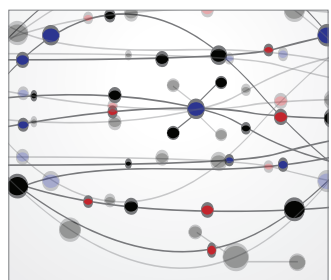

The Scientific World Journal
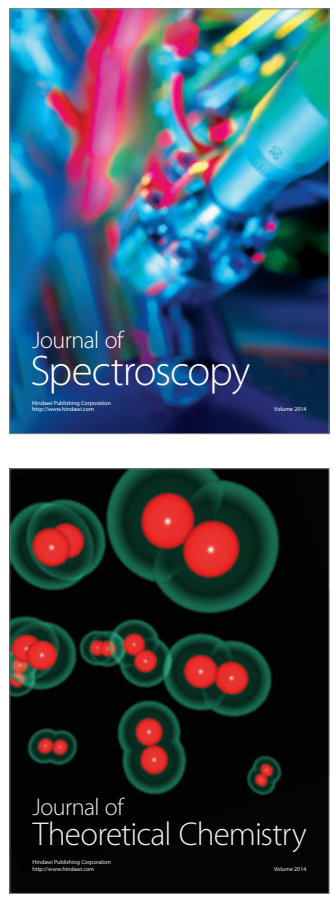
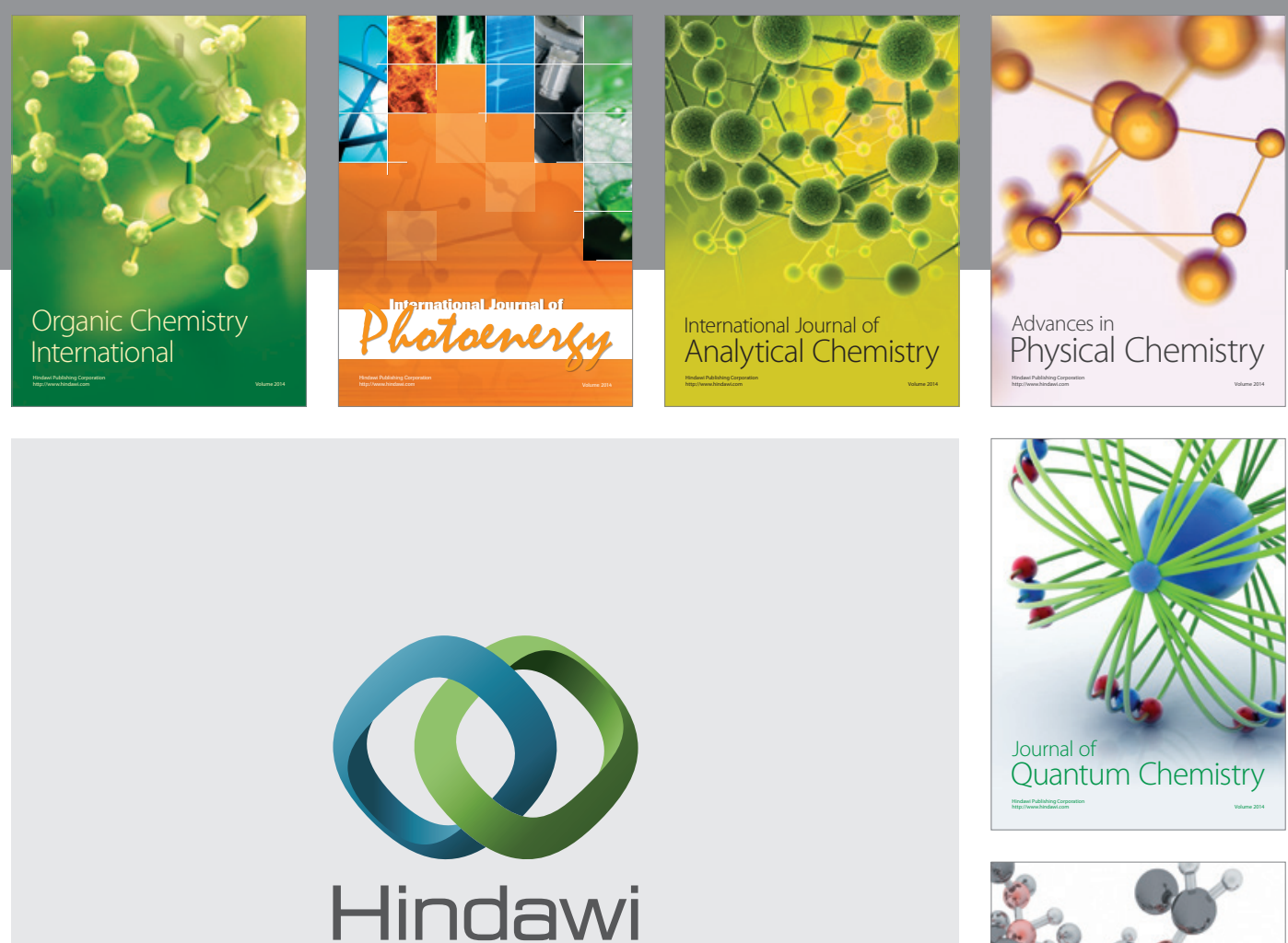

Submit your manuscripts at

http://www.hindawi.com

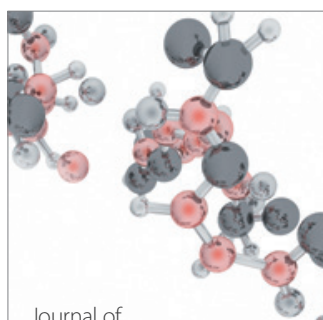

Analytical Methods

in Chemistry

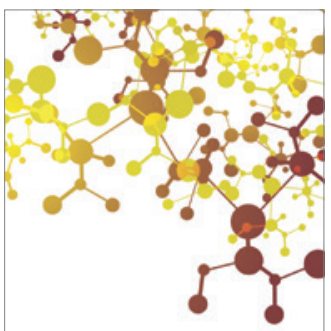

Journal of

Applied Chemistry

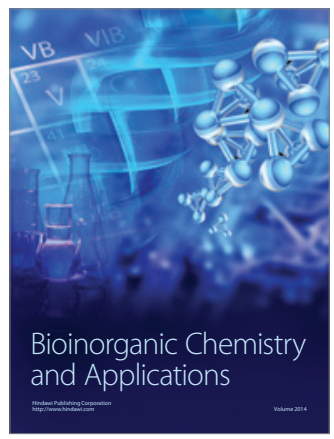

Inorganic Chemistry
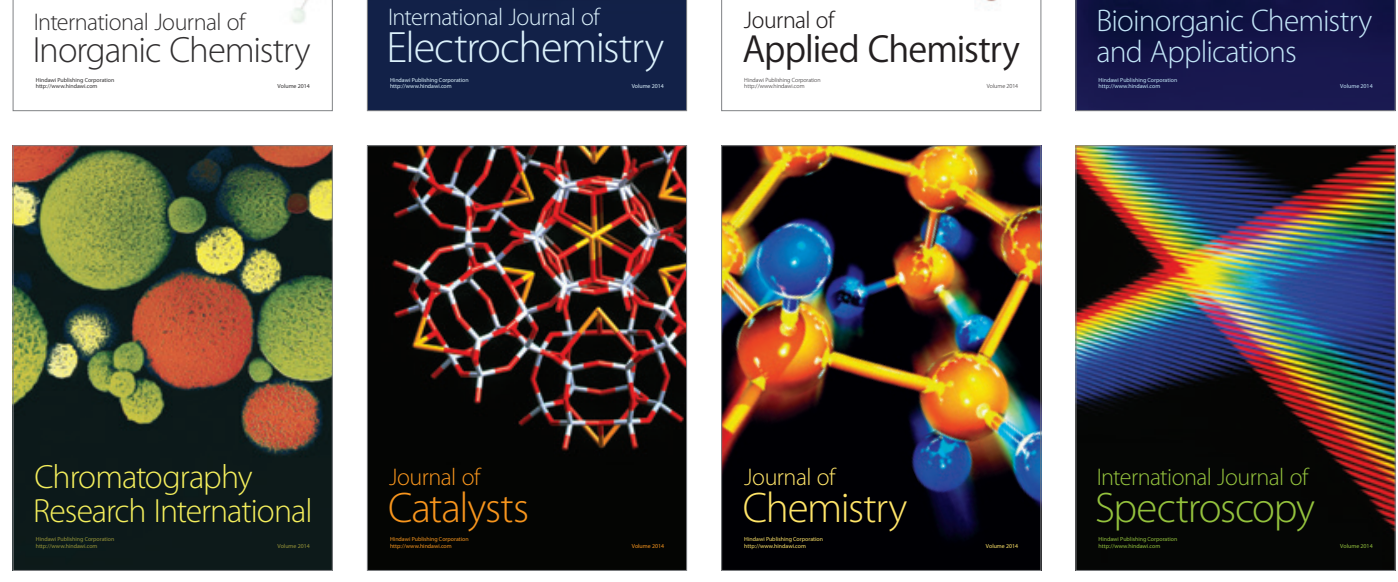